\title{
Communication \\ Effect of Spectral Signal-to-Noise Ratio on Resolution Enhancement at Surface Plasmon Resonance
}

\author{
Long Ma ${ }^{1}$, Guo Xia ${ }^{2,3,4, *}$, Shiqun Jin ${ }^{2,3,4}$, Lihao Bai ${ }^{1}$, Jiangtao Wang ${ }^{1}$, Qiaoqin Chen ${ }^{1}$ and Xiaobo Cai ${ }^{1}$ \\ 1 School of Instrument Science and Opto-Electronics Engineering, Hefei University of Technology, \\ Hefei 230009, China; malong@mail.hfut.edu.cn (L.M.); bailihao@mail.hfut.edu.cn (L.B.); \\ jiangtaowang@mail.hfut.edu.cn (J.W.); qqchen@mail.hfut.edu.cn (Q.C.); caixiaobo@mail.hfut.edu.cn (X.C.) \\ 2 Academy of Opto-Electric Technology, Hefei University of Technology, Hefei 230009, China; \\ shq_king@163.com \\ 3 Special Display and Imaging Technology Innovation Center of Anhui Province, School of Instrument Science \\ and Opto-Electronics Engineering, Hefei University of Technology, Hefei 230009, China \\ 4 National Engineering Laboratory of Special Display Technology, School of Instrument Science and \\ Opto-Electronics Engineering, Hefei University of Technology, Hefei 230009, China \\ * Correspondence: xiaguo@hfut.edu.cn
}

check for updates

Citation: Ma, L.; Xia, G.; Jin, S.; Bai, L.; Wang, J.; Chen, Q.; Cai, X. Effect of Spectral Signal-to-Noise Ratio on Resolution Enhancement at Surface Plasmon Resonance. Sensors 2021, 21 , 641. https://doi.org/10.3390/ s21020641

Received: 23 December 2020

Accepted: 14 January 2021

Published: 18 January 2021

Publisher's Note: MDPI stays neutral with regard to jurisdictional claims in published maps and institutional affiliations.

Copyright: (c) 2021 by the authors. Licensee MDPI, Basel, Switzerland. This article is an open access article distributed under the terms and conditions of the Creative Commons Attribution (CC BY) license (https:// creativecommons.org/licenses/by/ $4.0 /)$.

\begin{abstract}
Refractive index resolution is an important indicator for a wavelength interrogation surface plasmon resonance sensor, which can be affected by signal-to-noise ratio. This paper investigates the impact of spectral signal-to-noise ratio on a surface plasmon resonance sensor. The effects of different spectral powers and noises are compared and verified through simulation and experiments. The results indicate that the optimal resonance wavelength is changed and the refractive index resolution can even be nearly twice as good when the spectral signal-to-noise ratio is increased. The optimal resonance wavelength can be found by changing the spectral power distribution or noise.
\end{abstract}

Keywords: sensor; refractive index resolution; surface plasmon resonance; spectral signal-to-noise ratio

\section{Introduction}

Surface plasmon resonance (SPR) has been widely used in drug screening [1,2], biochemical safety [3], food safety [4], environmental monitoring [5], utilization of solar energy [6-8], and other fields $[9,10]$ in the last twenty years. SPR, which is an optical phenomenon characterized by excitation between the noble metal and the dielectric layer, has many merits, such as label-free real-time [11] monitoring, high sensitivity, and low cost. Meanwhile, the sensor can detect the sample regardless of whether the state of the sample is gas [12], liquid [13], or solid [14]. According to the interrogation method, the SPR system can be divided into angle interrogation [15], phase interrogation [16], amplitude interrogation [17], and wavelength interrogation [18]. The resolution of the wavelength-modulated SPR is changed with the wavelength. Therefore, finding the optimal resonance wavelength is an important basis to determine the working spectral band. However, the spectral signalto-noise ratio (SNR) can affect the determination of the optimal resonance wavelength.

Refractive index (RI) resolution has always been an important performance index. RI resolution is used to evaluate the smallest change of the resonance wavelength that can be distinguished in the SPR structure [19-21]. The RI resolution is defined as the ratio of the sensor's detection accuracy to sensitivity $[22,23]$. When the noise increases, the resonance wavelength fluctuates greatly. The changes of the spectral power distribution (SPD) can cause an asymmetrical change in the full width at half maximum (FWHM) of the SPR curve, and the asymmetry generates peak finding errors and increases the fluctuation of the resonance wavelength. Therefore, the spectral SNR [24] curve is a key factor affecting sensor performance. Vlek et al. changed the sensor layer structure and the metal layer structure to enhance the resolution of the SPR sensor [25]. Maharana et al. changed the prism material and metal layer material to enhance the resolution of the SPR sensor [15]. 
However, the methods, which improved the performance by changing the structure of the sensor, may increase the difficulty of production. Improving the resolution of the sensor without changing the structure and production of the sensor is a key research direction in the traditional Kretschmann structure. Chen et al. analyzed the influence of the resonance wavelength on the resolution of the sensor [22]. As a precursor of this paper, Zhou et al. analyzed the influence of three different SPDs on sensor resolution [26]. On the basis of these articles, this paper analyzes the influence of spectral SNR.

In this paper, the influence of spectral SNR on SPR is further studied based on the traditional Kretschmann structure. The SPD and noise are adjusted to obtain the optimal resonance wavelength, so that the working spectral band is then determined. In Section 2, we simulate the influence of the spectral SNR on the sensitivity, detection accuracy, and resolution by mathematical modeling. The influence of the spectral SNR is further obtained through physical experiments. In Section 3, the results of the simulation and the experiments are analyzed. Through the spectral SNR analysis, the optimal working spectral range of different types of sensors is obtained. Moreover, the influence of the spectral SNR on the best resolution and the best resonance wavelength is able to be evaluated.

\section{Materials and Methods}

\subsection{Transfer Matrix Modeling}

According to Maxwell's equations and boundary conditions [26,27], the reflectivity of the $N$-layer structure can be calculated by the transmission matrix method:

$$
M=\prod_{k=2}^{N-1} M_{k}=\left(\begin{array}{ll}
M_{11} & M_{12} \\
M_{21} & M_{22}
\end{array}\right)=\left(\begin{array}{cc}
\cos \beta_{k} & \frac{\left(-i \sin \beta_{k}\right)}{q_{k}} \\
\left(-i q_{k} \sin \beta_{k}\right) & \cos \beta_{k}
\end{array}\right),
$$

where $\beta_{k}=\left(\frac{2 \pi d_{k}}{\lambda}\right) \times\left(\varepsilon_{k}-n_{1}^{2} \sin \theta_{1}\right)^{\frac{1}{2}}, q_{k}=\frac{\left(\varepsilon_{k}{ }^{2}-n_{1}{ }^{2} \sin \theta_{1}\right)^{\frac{1}{2}}}{\varepsilon_{k}}, d_{k}$ represents the $k_{t h}$ layer thickness, $n_{k}$ represents the RI in the $k_{t h}$ layer, and $\theta_{1}$ represents the incident angle.

$$
R_{p}=\left(\phi_{p}\right)^{2}=\left[\frac{\left(M_{11}+M_{12} q_{N}\right) q_{1}-\left(M_{21}+M_{22} q_{N}\right)}{\left(M_{11}+M_{12} q_{N}\right) q_{1}+\left(M_{21}+M_{22} q_{N}\right)}\right]^{2}
$$

where $R_{p}$ is the reflectance. The incident angles $\theta_{1}$ are $42.6^{\circ}, 42.7^{\circ}, 42.75^{\circ}, 42.8^{\circ}, 43^{\circ}, 43.3^{\circ}$, $43.5^{\circ}, 43.7^{\circ}, 44^{\circ}$, and $44.6^{\circ}$. The parameter values in the mathematical model will be listed below.

\subsection{Model Parameters}

In Figure $1, n_{B K 7}$ can be calculated as follows [26,28]:

$$
n_{B K 7}=\left(\frac{1.03961212 \lambda^{2}}{\lambda^{2}-0.00600069867}+\frac{0.231792344 \lambda^{2}}{\lambda^{2}-0.0200179144}+\frac{1.03961212 \lambda^{2}}{\lambda^{2}-103.560653}\right)^{\frac{1}{2}},
$$

According to the Drude-Lorentz model, the thickness of the gold film is $50 \mathrm{~nm}$ and the dielectric constant can be calculated as follows [26]:

$$
\varepsilon_{m}=\left[1-\frac{\lambda_{c} \lambda^{2}}{\lambda_{p}^{2}\left(\lambda_{c}+i \lambda\right)}\right]
$$

where $\lambda_{p}=168.26 \mathrm{~nm}$ is the plasma wavelength and $\lambda_{c}=8934.2 \mathrm{~nm}$ is the collision wavelength.

The three kinds of SPD are simulated in the sensor simulation. 


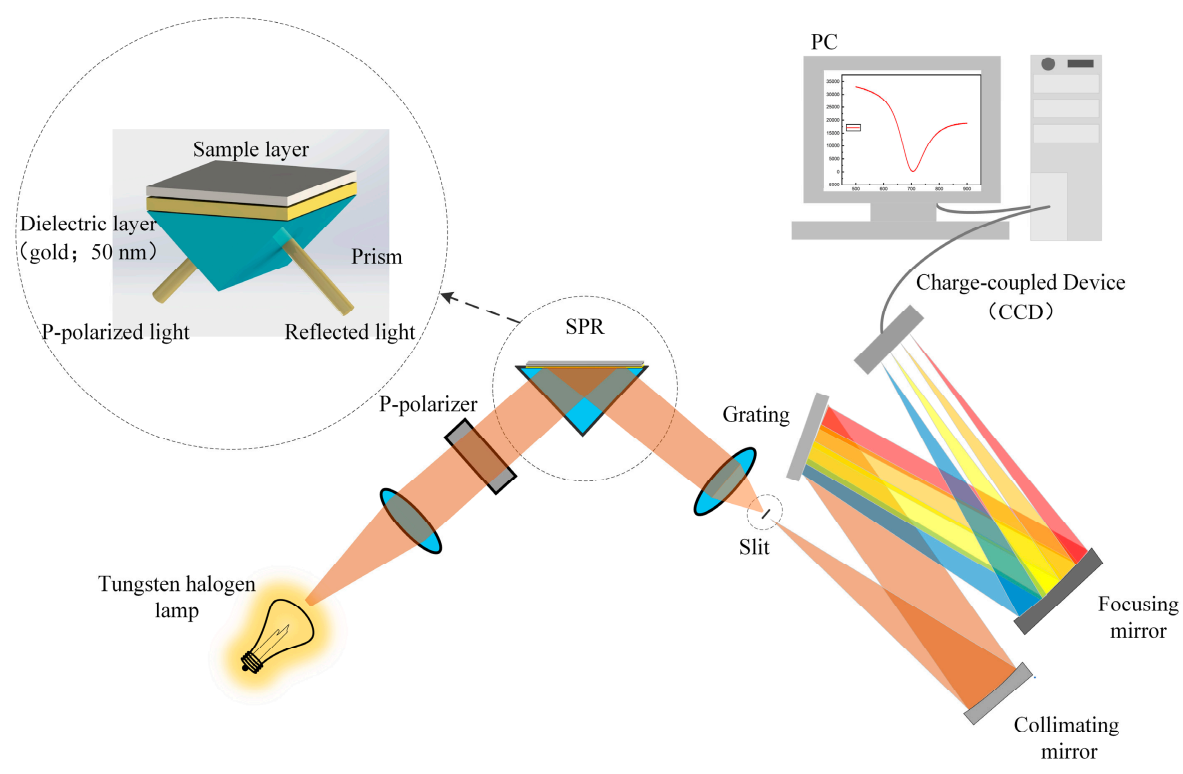

Figure 1. Schematic of a surface plasmon resonance (SPR) sensor.

As shown in Figure 2, among the three SPDs, SPD1 is the ideal light source, and SPD2 and SPD3 change the slope of the SPD to obtain different changing trends of spectral SNR and performance indicators.

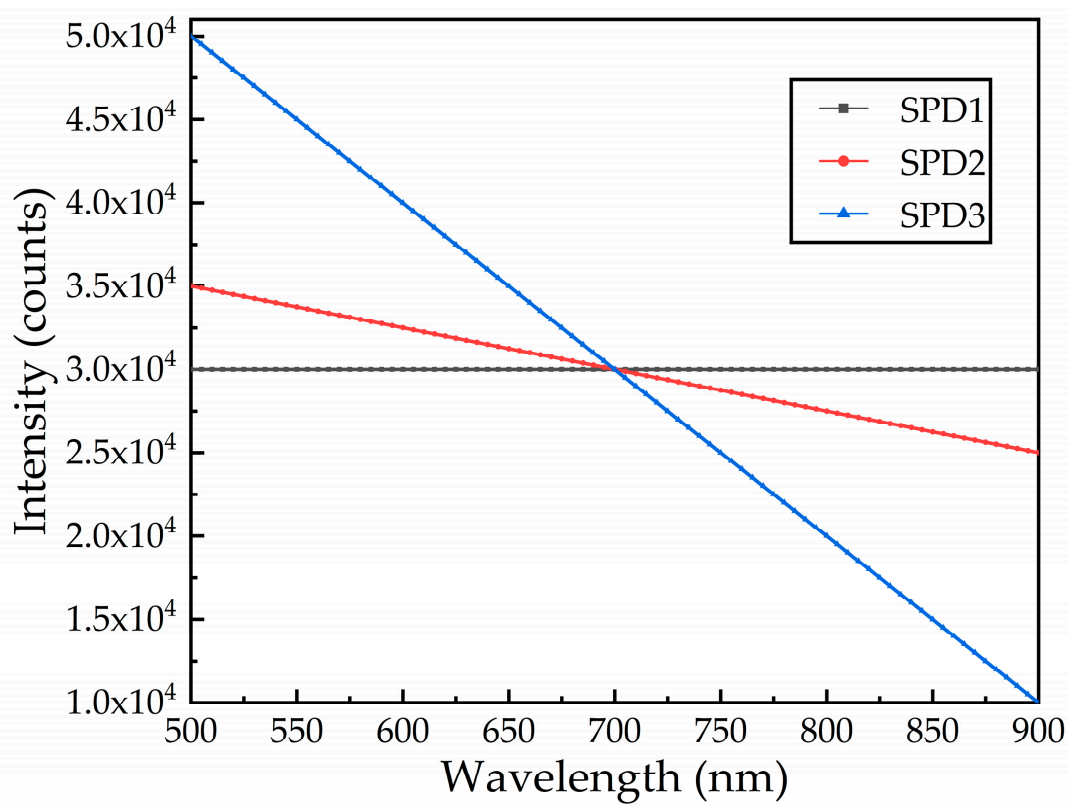

Figure 2. Simulate three spectral power distribution (SPD) curves [26].

In the actual measurements, the uncontrollable noise mainly comes from the detector, which is mainly divided into the readout noise, dark noise, fixed mode noise, and photoelectric noise [22,29]. Each noise is independent, and the total noise can be expressed as follows:

$$
N=\sqrt{N_{R}^{2}+N_{D}^{2}+N_{P}^{2}+N_{F}^{2}},
$$

As shown in Figure 3, the noise simulation value is given separately, and the final calculated simulation value $N$ is given. $N_{R}$ is dependent on the circuit design of the instrument and is mainly generated when the analog signal is transformed into a digital signal. $N_{R}$ can be calculated as follows [29]: 


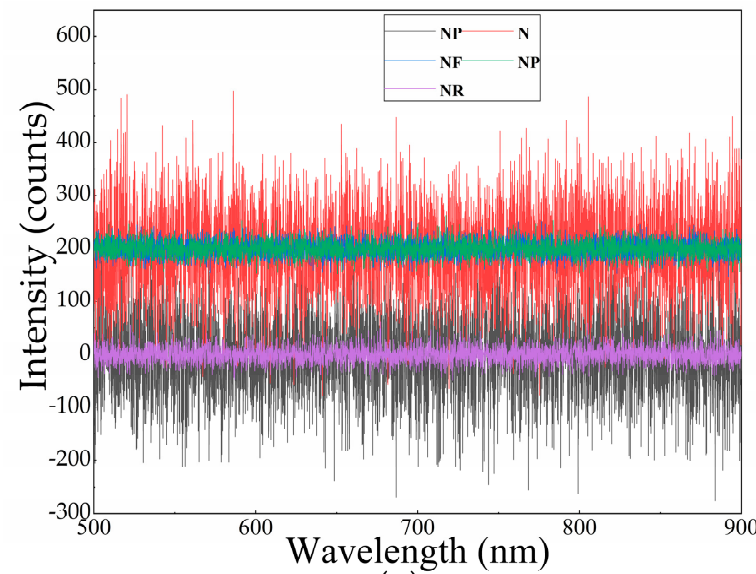

(a)

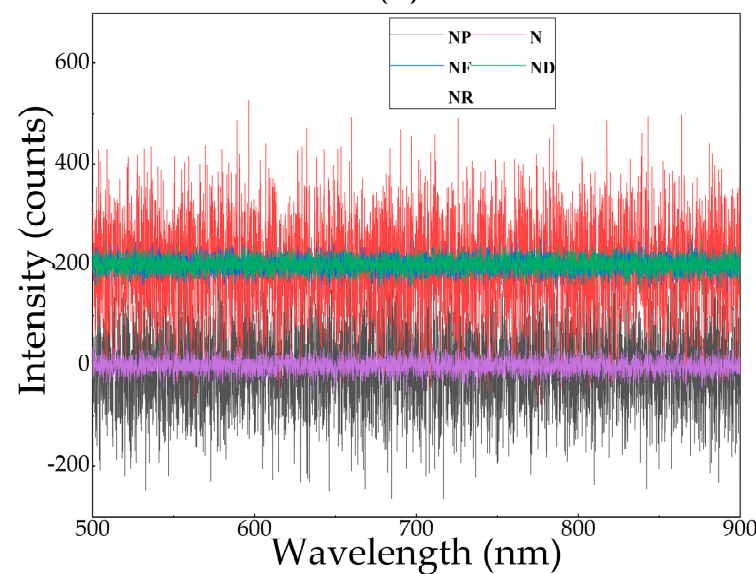

(c)

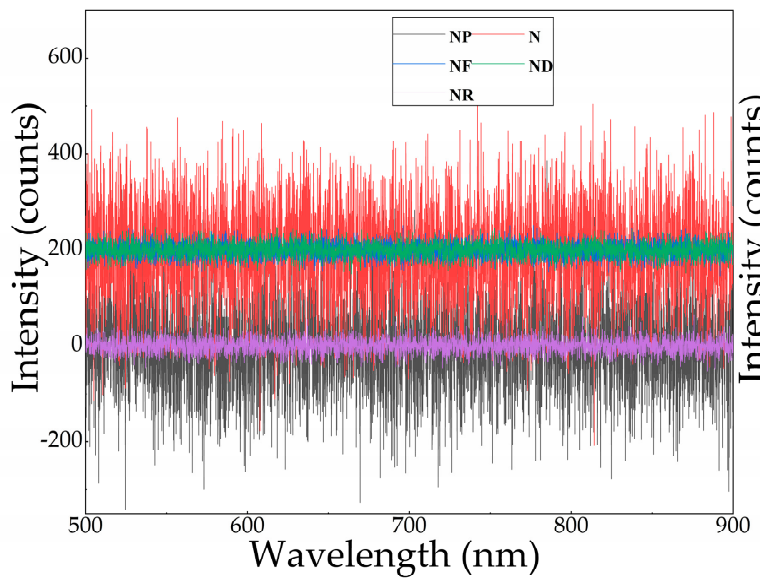

(e)

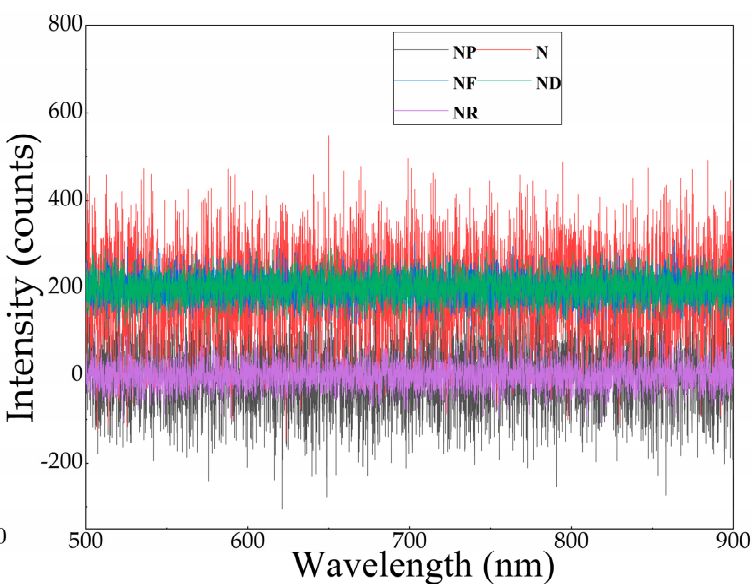

(b)

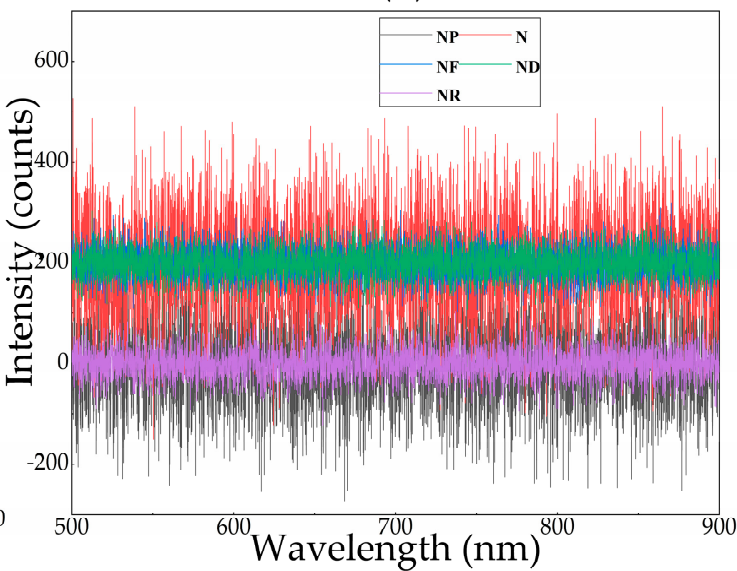

(d)

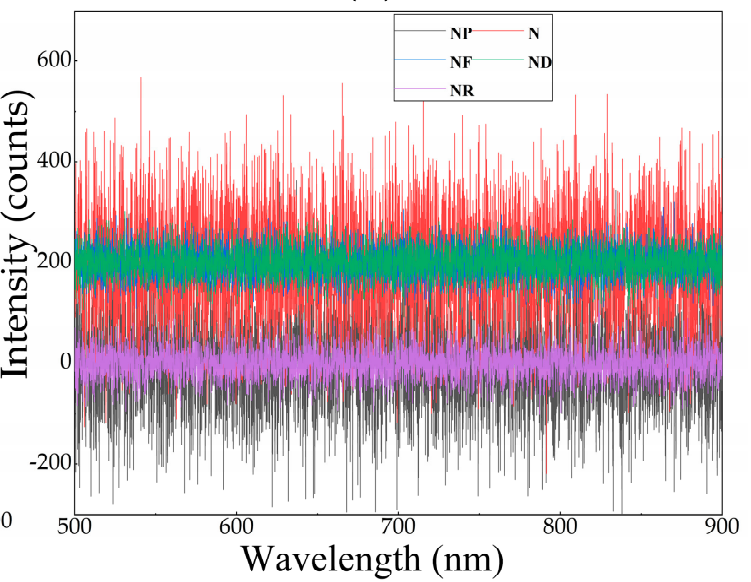

(f)

Figure 3. Noise value in simulation: (a) noise level (under SPD1), (b) double noise level (under SPD1), (c) noise level (under SPD2), (d) double noise level (under SPD2), (e) noise level (under SPD3), and (f) double noise level (under SPD3). $N$ is the final calculated simulation value; $N_{p}$ is the photoelectron noise; $N_{F}$ is the fixed mode noise caused by the difference in pixel dark current; $N_{D}$ is the dark noise in the detector; and $N_{R}$ is dependent on the circuit design of the instrument.

$$
N_{R}=\operatorname{std}\left(S_{\text {bias }}\right)
$$


$N_{D}$ is the dark noise in the detector because of the thermal movement of particles, which produces current at the output end. $N_{D}$ can be calculated as follows [29]:

$$
N_{D}=\operatorname{std}\left(S_{D}\right),
$$

$N_{F}$ is the fixed mode noise caused by the difference in pixel dark current, which is mainly determined by the manufacturing process. $N_{F}$ can be calculated as follows [29]:

$$
N_{F}=S_{F},
$$

$N_{p}$ is the photoelectron noise, which is determined by the statistical difference of particle arrival at the detector. $N_{p}$ can be calculated as follows [29]:

$$
N_{P}=\frac{\sqrt{n_{e-}}}{g}
$$

where $S_{\text {bias }}$ is a bias signal, $S_{D}$ is the dark current signal and wavelength point at the current wavelength point, $n_{e-}$ is the number of detected photoelectrons, and $g$ is gain constant. Given the influence of total noise, the SPR measurement curve is expressed as follows [26]:

$$
S_{m}=R_{p} \times S P D+N,
$$

where $S_{m}$ is the measurement curve, $R_{p}$ is the reflectance curve, and $N$ is the total noise. This paper qualitatively analyses the effects of spectral SNR through the spectral SPR curve. During the simulation, the results are changed by adjusting the noise and SPD. The RI resolution is used as an evaluation index to judge the influence of the spectral SNR.

Three different SPDs are constructed and two noise levels are added. The standard deviation of resonance wavelength is regarded as noise for each resonance wavelength. The light intensity value is used as the signal value in the current wavelength. The spectral SNR corresponding to the resonance wavelength is simulated, as is shown in Figure 4a,d,g. The spectral SNR is calculated as follows:

$$
S N R=10 \times \log _{10}\left(\operatorname{abs}\left(\frac{P_{s}}{P_{n}}\right)\right),
$$

$P_{S}$ is the spectral power signal, and $P_{n}$ represents the resonant wavelength noise.

Figure 4 shows the spectral SNRs and fitted SPR curves under three spectral powers along with two types of noise. Spectral SNRs show the same trend as SPD, and the fitted SPR curves of the spectral power under different wavelengths are given. The fitted SPR curves are changed under different spectral SNRs. In the next part, we analyze the changes in sensor performance based on the changes in the fitted curves.

\subsection{RI Resolution}

The resolution formula of RI can be obtained as follows [30]:

$$
\delta_{n}=\frac{\delta_{\lambda}}{S_{n}},
$$

where $\delta_{n}, \delta_{\lambda}$, and $S_{n}$ are the RI resolution, RI detection accuracy, and RI sensitivity of the SPR sensor, respectively [31]. $S_{n}$ is an important indicator of the sensor's static characteristics and is defined as the ratio of the output change to the input change. In the SPR sensor, when the RI of the sample changes, the resonance wavelength $\lambda$ also changes. The RI sensitivity can be calculated as follows:

$$
S_{n}=\frac{\Delta \lambda}{\Delta n},
$$



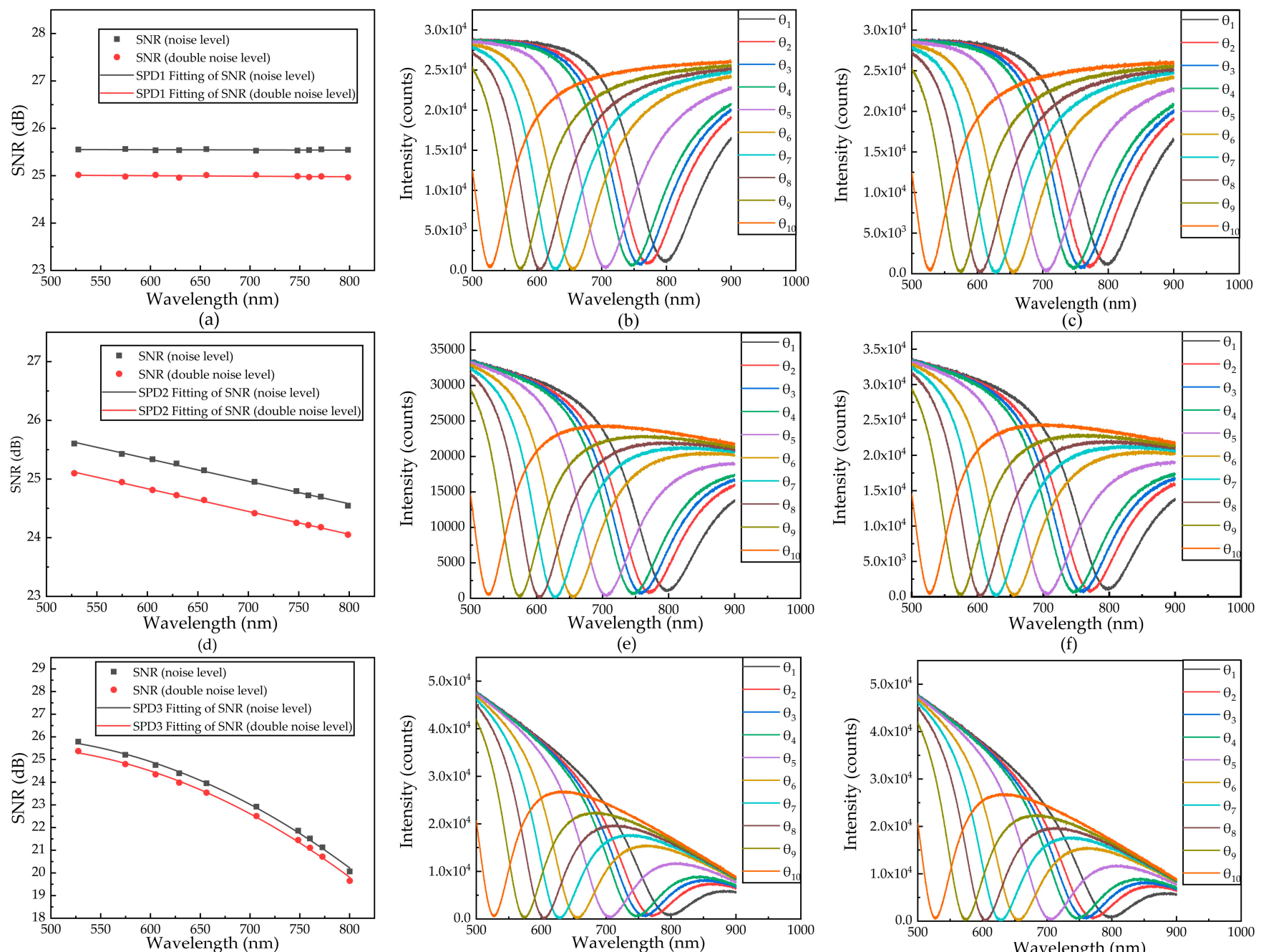

(g)

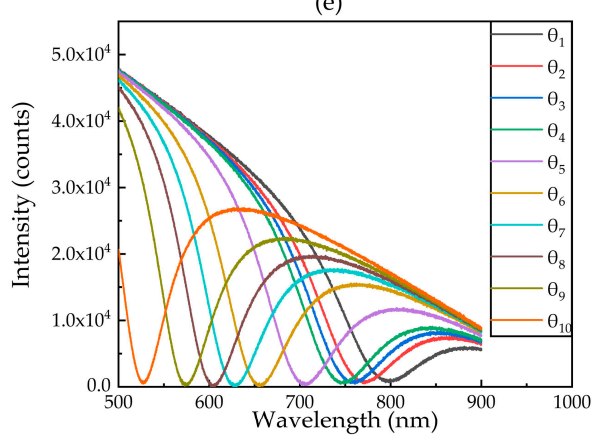

(h)

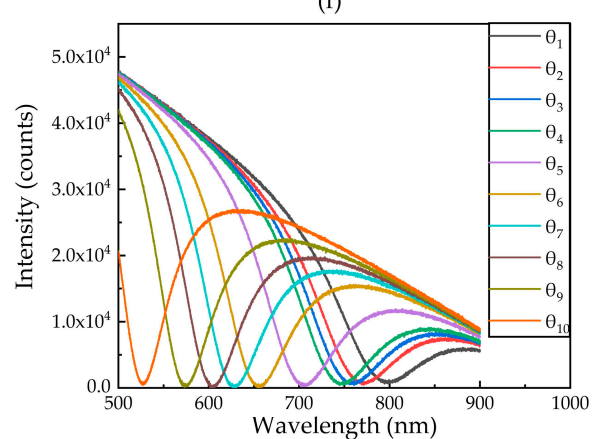

(i)

Figure 4. (a) Simulation of the spectral SNR fitting curve under two noise levels and SPD1. (b) Fitted SPR curve with a double noise level (under SPD1). (c) Fitted SPR curve with a noise level (under SPD1). (d) Simulation of the spectral SNR fitting curve under two noise levels and SPD2. (e) Fitted SPR curve with a double noise level (under SPD2). (f) Fitted SPR curve with a noise level (under SPD2). (g) Simulation of the spectral SNR fitting curve under two noise levels and SPD3. (h) Fitted SPR curve with a double noise level (under SPD3). (i) Fitted SPR curve with a noise level (under SPD3).

The RI of the sample is from 1 to $1+\Delta n(\Delta n=0.00001)$, the deviation of resonance wavelength is obtained, and the sensitivity of the sensor is calculated and fitted by Equation (13). The fitted results are shown in Figure 5.

As shown in Figure 5, the sensitivity increases with resonance wavelength. The trend between resonance wavelength and sensor sensitivity under different conditions is the same. The increase in sensitivity with wavelength is consistent with the results obtained by mathematical model analysis [30]. There is a slight difference in sensitivity, which is mainly due to the influence of the spectral SNR on the wavelength accuracy in the peak seeking process. The difference in the SPD results in the shift of the resonance wavelength, thus the sensitivity is affected. 


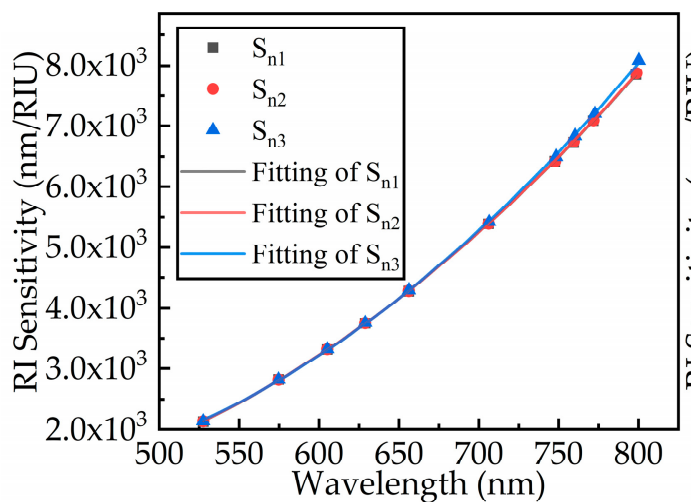

(a)

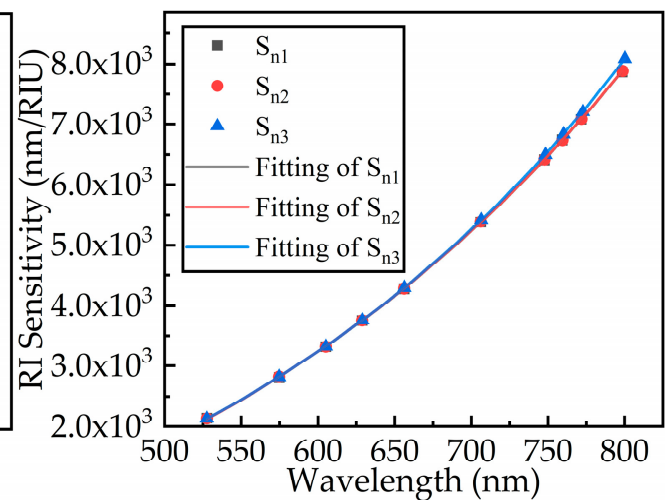

(b)

Figure 5. Three signal refractive index (RI) sensitivity curves for (a) noise level and (b) double noise level.

$\delta_{\lambda}$ is mainly affected by the FWHM and minimum value of the SPR curve. To verify this result, the effects of three trending spectral SNRs are simulated. The resonance wavelength corresponding to each angle is simulated 1000 times, and the standard deviation of the resonance wavelength here is used as the detection accuracy of the current wavelength.

As displayed in Figure 6a,b, the growth rate in the resonance wavelength of 500-650 nm is small, indicating that the resonance wavelength value is relatively stable. When the resonance wavelength is $650-800 \mathrm{~nm}$, as the resonance wavelength increases, the detection accuracy growth rate increases violently. Because the spectral SNR is very small, the fluctuation of the detected resonance wavelength changes violently, which leads to an increase in the standard deviation of the resonance wavelength and an obvious decrease in system performance. The results show that the spectral SNR can affect the detection accuracy of the current resonance wavelength.

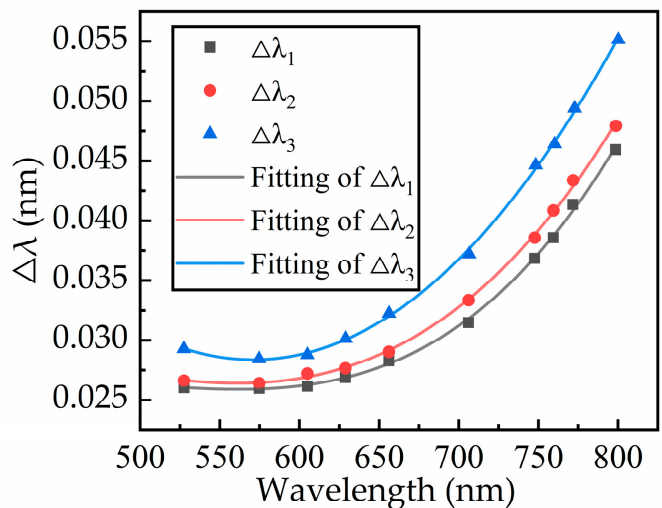

(a)

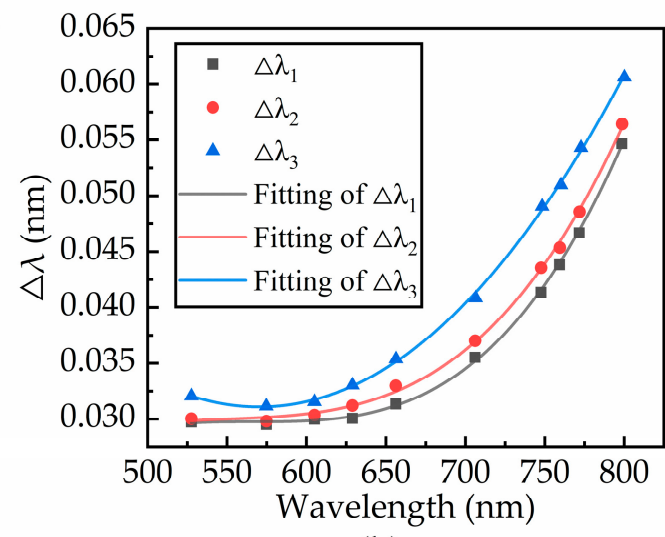

(b)

Figure 6. Three signal standard deviation curves for (a) noise level and (b) double noise level.

In the simulation, three spectral power curves and two noise levels are presented. The noise and SPD are combined to obtain six simulation results. The influence of the spectral SNR is obtained through methods of controlling variables comparison.

\subsubsection{Same Noise Levels at Different SPDs}

In this case, the changing trend of resolution is obtained by simulating the three SPDs under the same noise level. Figure 7 shows the effect of noise on RI resolution. 


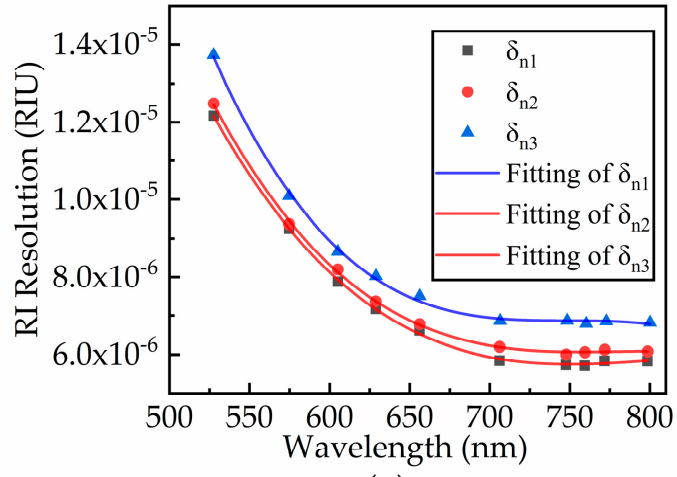

(a)

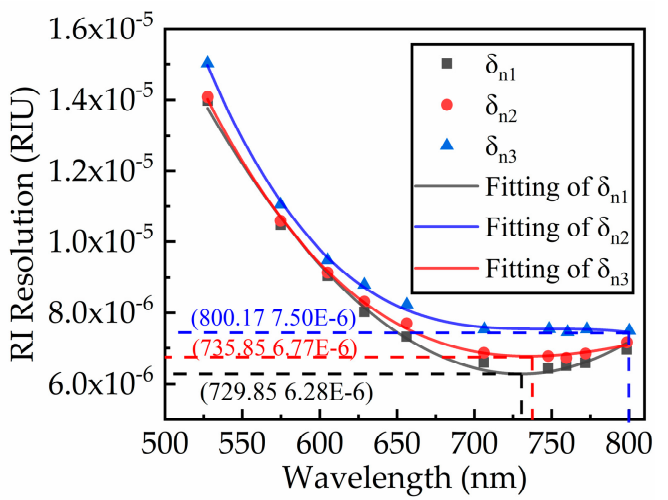

(b)

Figure 7. Three signal RI resolution curves for (a) noise level and (b) double noise level.

As shown in Figure 7, when the resonance wavelength is $500-650 \mathrm{~nm}$, the resolution of the system changes monotonically. When the resonance wavelength is $650-800 \mathrm{~nm}$, the resolution of the system presents different trends in two cases. The reason for the phenomena is that the spectral SNR of the system decreases rapidly, which means the noise will cause more impacts on the system. The impacts are so severe that the system cannot maintain its own modulated mode, resulting in a different trend of the resolution changes. Thus, it is important to find the optimal resonance wavelength. The optimal resonance wavelength is obtained by changing the SPD.

\subsubsection{Same SPD at Different Noise Levels}

When the SPD is kept constant, the influence of noise on the sensor resolution can be observed. The influence of different noise levels on the current resonance wavelength and the best resolution is given in Figure 8. The effect of the different noise levels at the same SPD is obtained.

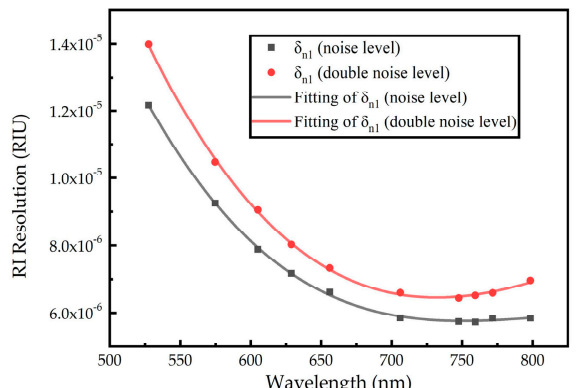

(a)

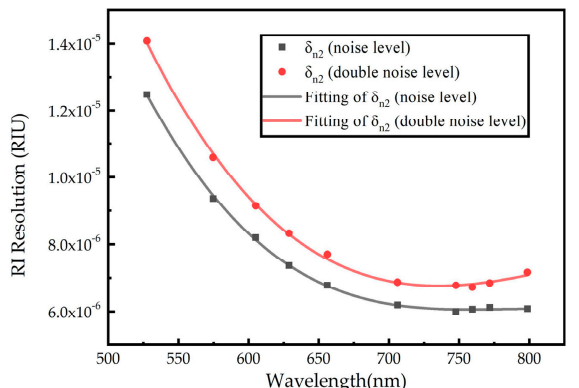

(b)

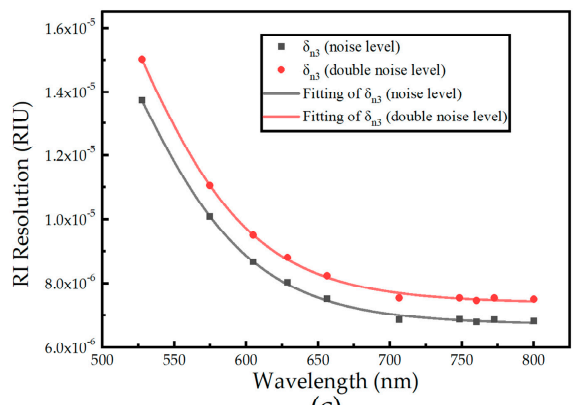

(c)

Figure 8. Resolution fitting curve of two noise levels (a) under SPD1; (b) under SPD2; and (c) under SPD3.

\subsection{Experimental}

The system consists of a light source (halogen lamp, Daheng Optoelectronic Technology Co., Ltd., beijing, China), a collimator ( $\Phi 5.0 \mathrm{~mm}$, SMA905, Daheng Optoelectronic Technology Co., Ltd., beijing, China), a polarizer ( $\$ 25.4$ mm, FU-PZP-Y24, Daheng Optoelectronic Technology Co., Ltd., beijing, China), a SPR device (right-angle prism BK7, Daheng Optoelectronic Technology Co., Ltd., beijing, China, gold film thickness $50 \mathrm{~nm}$ ), and a spectrometer (USB4000+ Ocean Optics, Weihai Optical Instrument Co., Ltd., Shanghai, China). The spectral curve of the halogen tungsten lamp is unstable for a short period, which affects the accuracy of the sensor experiment. The measurement experiment can be carried out when the measured spectral curve does not show a large jump. The light source is warmed up for $5 \mathrm{~min}$ and the measurement begins when the light source is stable. The 
measurement needs to be stopped after a period of measurement to prevent the stability of the light source from deteriorating. In the next part, the experimental results are described.

Figure 9a shows the spectral curves of different integration times from the same spectrometer. The fitted spectral SNR curve for the spectral curves of different integration times is shown in Figure 9b. The measured SPR curves of the two spectral signals after adjusting the integration time in the SPR device are shown in Figure 9c,d.
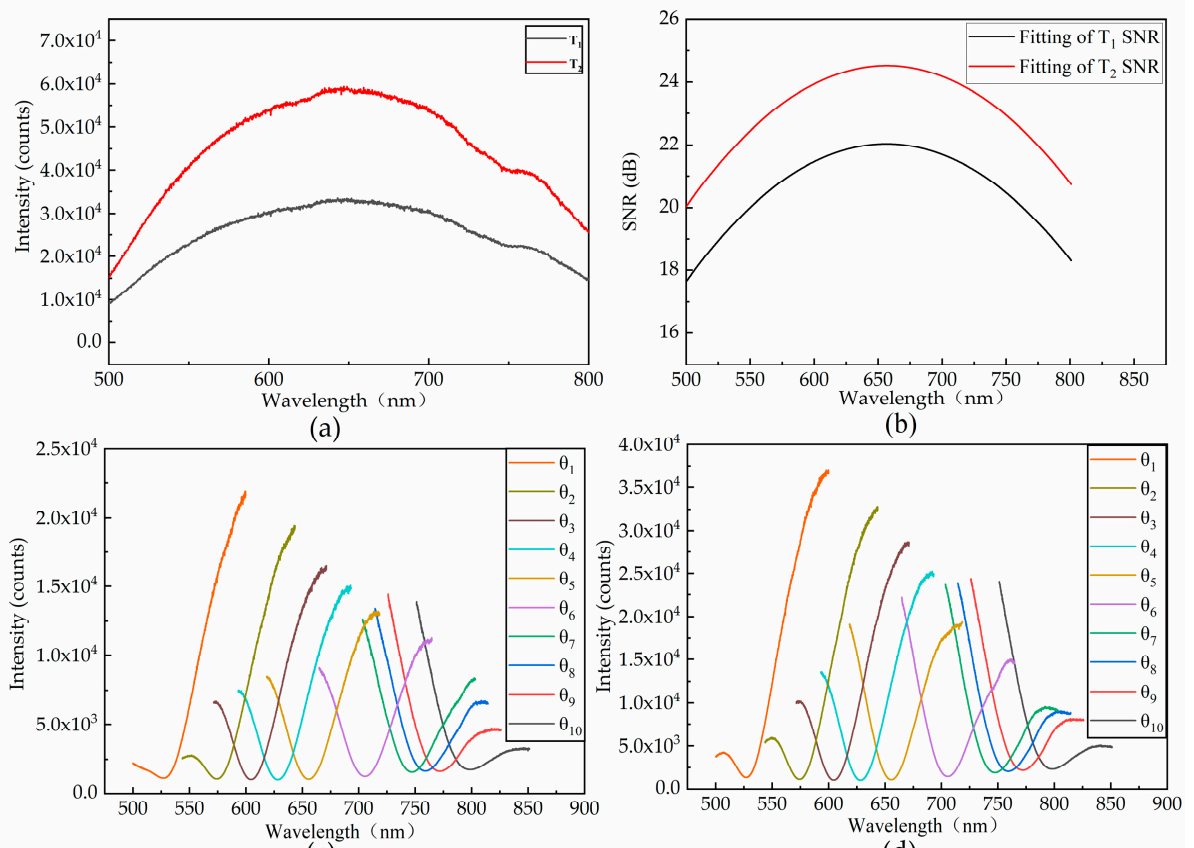

(c)

(d)

Figure 9. (a) SPD curves received at two different integration times. (b) Fitting of the spectral SNR curve under two different integration times: (c) integration time T1 (SPR curve); (d) integration time T2 (SPR curve).

\section{Results and Discussion}

\subsection{Simulation Results}

As shown in Table 1, when the noise level doubles, the spectral SNR of the same SPD decreases, and the optimal resonance wavelength decreases. When the signal value is SPD3, the optimal resonance wavelength increases with the noise level. There are two reasons for this situation. On the one hand, due to the limitation of the measurement range, the maximum resonance wavelength of the measurement is around $800 \mathrm{~nm}$ (as shown in Figure 4). On the other hand, the light intensity is weak, and the optimal resonance wavelength is around $800 \mathrm{~nm}$, which is the change caused by noise (as shown in Figure 8c). As the spectral SNR decreases, the numerical of the resolution increases, which means a decrease in resolution.

Table 1. SPR resolution simulation results.

\begin{tabular}{cccc}
\hline & $\begin{array}{c}\text { Spectral Power } \\
\text { Distribution }\end{array}$ & $\begin{array}{c}\text { Optimal Resonance } \\
\text { Wavelength }(\mathbf{n m})\end{array}$ & Best RI Resolution (RIU) \\
\hline \multirow{3}{*}{ Noise level } & SPD1 & 759.30 & $5.72 \times 10^{-6}$ \\
& SPD2 & 795.15 & $6.08 \times 10^{-6}$ \\
& SPD3 & 798.23 & $6.78 \times 10^{-6}$ \\
\hline \multirow{5}{*}{ Double Noise level } & SPD1 & 729.85 & $6.28 \times 10^{-6}$ \\
& SPD2 & 735.85 & $6.77 \times 10^{-6}$ \\
& SPD3 & 800.17 & $7.50 \times 10^{-6}$ \\
\hline
\end{tabular}




\subsection{Experimental Results}

In the experiment, the RI of the sample was changed at equal intervals and changes in the spectral curve were recorded 1000 times. Based on the data obtained from the experimental operations above, the sensitivity and detection accuracy of the current resonance wavelength was calculated, and the current resonance wavelength resolution was modeled in Equation (12), as shown in Figure 10.

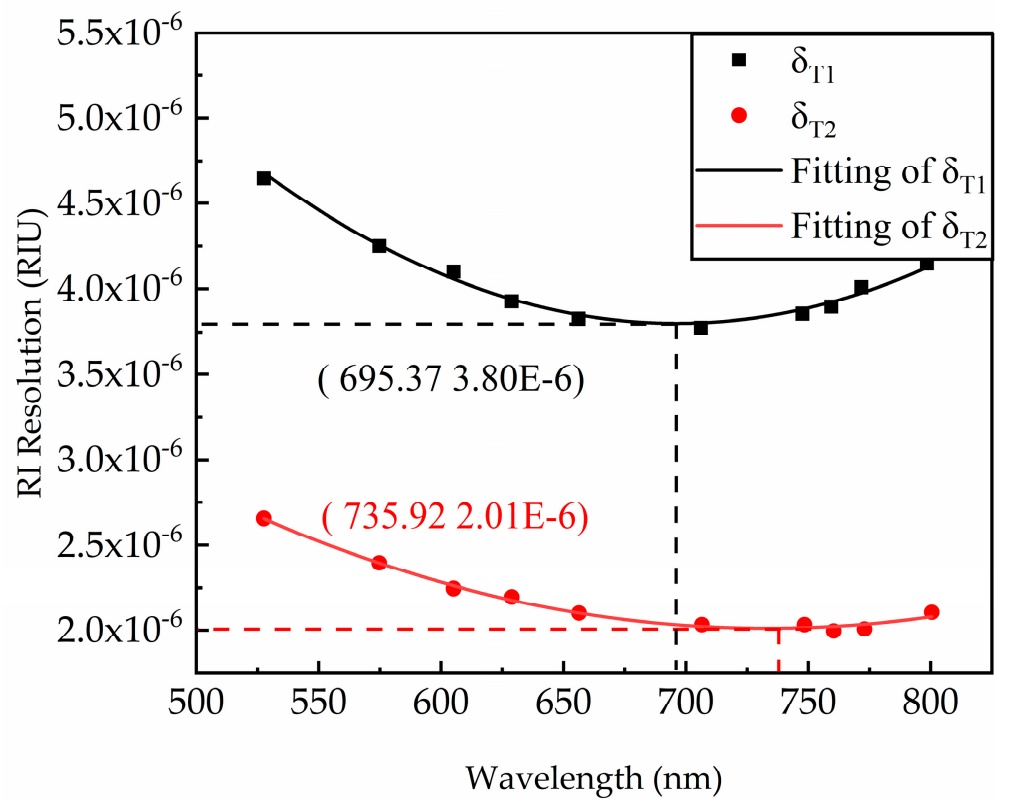

Figure 10. The optimal resonance wavelength at the integration time T1 (integration time $8 \mathrm{~ms}$ ) is $695.37 \mathrm{~nm}$, and the best RI resolution is $3.80 \times 10^{-6} \mathrm{RIU}$. When the integration time is T2 (integration time $15 \mathrm{~ms}$ ), the optimal resonance wavelength is $735.92 \mathrm{~nm}$, and the best RI resolution is $2.01 \times 10^{-6} \mathrm{RIU}$.

\subsection{Discussion}

The spectral SNR was changed under different light source wavelengths to determine the performance index of the SPR device. The optimal resonance wavelength was found, and the measurement was taken near the optimal resonance wavelength position to improve the reliability and accuracy of the results. The relationship between spectral SNR and RI resolution between replacement device components and different types of devices may be different from the results of this experiment, but the overall trend should be the same. This experiment provides a technical reference for the performance evaluation of different systems of wavelength-interrogated SPR.

\section{Conclusions}

The spectral SNR obviously affects the optimal resonance wavelength and the optimal resolution. In the experiment, the optimal resonance wavelength and the optimal resolution were obtained at two integration times. On the spectral power curve, the effect of the spectral SNR on the resonance wavelength could be obtained through experiments. The influence in the change of integration time on the spectral SNR is shown in Figure $9 \mathrm{~b}$. With the extension of the integration time (T1-T2), the spectral SNR increases, and the optimal resonance wavelength of the sensor increases. Meanwhile, the numerical resolution decreases, which means an increase in resolution. The practicality of surface plasmon resonance devices are greatly improved because the spectral SNR is a common physical quantity for different coupling and sensing layer structures. The replacement of components in the system may cause changes in the measurement results. Therefore, each replacement of components requires recalibration of the SPR equipment. 
Author Contributions: Conceptualization: G.X.; Methodology: G.X. and L.M.; Validation: S.J.; Data curation: L.B., J.W. and Q.C.; Writing—original draft preparation: L.M.; Writing—review and editing: L.M. and X.C.; Funding acquisition: G.X. All authors have read and agreed to the published version of the manuscript.

Funding: This research was funded by Key Research and Development Program of Anhui Province, grant number 1804d08020310.

Institutional Review Board Statement: Not applicable.

Informed Consent Statement: Not applicable.

Data Availability Statement: Not applicable.

Conflicts of Interest: The authors declare no conflict of interest.

\section{References}

1. Loo, F.C.; Ng, S.P.; Wu, C.M.L.; Kong, S.K. An aptasensor using DNA aptamer and white light common-path SPR spectral interferometry to detect cytochrome-c for anti-cancer drug screening. Sens. Actuators B: Chem. 2014, 198, 416-423. [CrossRef]

2. Chung, J.W.; Kim, S.D.; Bernhardt, R.; Pyun, J.C. Application of SPR biosensor for medical diagnostics of human hepatitis B virus (hHBV). Sens. Actuators B: Chem. 2005, 111, 416-422. [CrossRef]

3. Rasooly, A. Surface plasmon resonance analysis of staphylococcal enterotoxin B in food. J. Food Prot. 2001, 64, 37-43. [CrossRef] [PubMed]

4. Conteduca, D.; Dell'Olio, F.; Innone, F.; Ciminelli, C.; Armenise, M.N. Rigorous design of an ultra-high Q/V photonic/plasmonic cavity to be used in biosensing applications. Opt. Laser Technol. 2016, 77, 151-161. [CrossRef]

5. Hu, C.; Gan, N.; Chen, Y.; Bi, L.; Zhang, X.; Song, L. Detection of microcystins in environmental samples using surface plasmon resonance biosensor. Talanta 2009, 80, 407-410. [CrossRef] [PubMed]

6. Yu, P.Q.; Yang, H.; Chen, X.F.; Yi, Z.; Yao, W.T.; Chen, J.F.; Yi, Y.G.; Wu, P.H. Ultra-wideband solar absorber based on refractory titanium metal. Renew. Energy 2020, 158, 227-235. [CrossRef]

7. Yi, Z.; Li, J.K.; Lin, J.C.; Qin, F.; Chen, X.F.; Yao, W.T.; Liu, Z.M.; Cheng, S.B.; Wu, P.H.; Li, H.L. Broadband polarization-insensitive and wide-angle solar energy absorber based on tungsten ring-disc array. Nanoscale 2020, 12, 23077-23083. [CrossRef]

8. Li, J.K.; Chen, X.F.; Yi, Z.; Yang, H.; Tang, Y.J.; Yi, Y.; Yao, W.T.; Wang, J.Q.; Yi, Y.G. Broadband solar energy absorber based on monolayer molybdenum disulfide using tungsten elliptical arrays. Mater. Today Energy 2020, 16, 100390. [CrossRef]

9. Chu, P.X.; Chen, J.X.; Xiong, Z.G.; Yi, Z. Controllable frequency conversion in the coupled time-modulated cavities with phase delay. Opt. Commun. 2020, 476, 126338. [CrossRef]

10. Zhang, Y.B.; Wu, P.H.; Zhou, Z.G. Study on Temperature Adjustable Terahertz Metamaterial Absorber Based on Vanadium Dioxide. IEEE Access 2020, 8, 85154-85161. [CrossRef]

11. Karamanska, R.; Clarke, J.; Blixt, O.; MacRae, J.I.; Zhang, J.Q.; Crocker, P.R.; Laurent, N.; Wright, A.; Flitsch, S.L.; Russell, D.A.; et al. Surface plasmon resonance imaging for real-time, label-free analysis of protein interactions with carbohydrate microarrays. Glycoconj. J. 2008, 25, 69-74. [CrossRef] [PubMed]

12. Liedberg, B.; Nylander, C.; Lunström, I. Surface plasmon resonance for gas detection and biosensing. Sens. Actuators 1983, 4, 299-304. [CrossRef]

13. Zhao, Y.; Deng, Z.; Wang, Q. Fiber optic SPR sensor for liquid concentration measurement. Sens. Actuators B: Chem. 2014, 192, 229-233. [CrossRef]

14. Mivehi, L.; Bordes, R.; Holmberg, K. Adsorption of cationic gemini surfactants at solid surfaces studied by QCM-D and SPR: Effect of the rigidity of the spacer. Langmuir 2011, 27, 7549-7557. [CrossRef] [PubMed]

15. Maharana, P.K.; Jha, R. Chalcogenide prism and graphene multilayer based surface plasmon resonance affinity biosensor for high performance. Sens. Actuators B: Chem. 2012, 169, 161-166. [CrossRef]

16. Chiang, H.P.; Lin, J.L.; Chen, Z.W. High sensitivity surface plasmon resonance sensor based on phase interrogation at optimal incident wavelengths. Appl. Phys. Lett. 2006, 88, 141105. [CrossRef]

17. Piliarik, M.; Homola, J. Surface plasmon resonance (SPR) sensors: Approaching their limits? Opt. Express 2009, 17, 16505-16517. [CrossRef]

18. Yi, S.J.; Yuk, J.S.; Jung, S.H.; Zhavnerko, G.K.; Kim, Y.M.; Ha, K.S. Investigation of selective protein immobilization on charged protein array by wavelength interrogation-based SPR sensor. Mol. Cells 2003, 15, 333.

19. Jiang, L.Y.; Yuan, C.; Li, Z.Y.; Su, J.; Yi, Z.; Yao, W.T.; Wu, P.H.; Liu, Z.M.; Cheng, S.B.; Pan, M. Multi-band and high-sensitivity perfect absorber based on monolayer grapheme metamaterial. Diam. Relat. Mater. 2021, 111, 108227. [CrossRef]

20. Zhang, Y.B.; Yi, Z.; Wang, X.Y.; Chu, P.X.; Yao, W.T.; Zhou, Z.G.; Cheng, S.B.; Liu, Z.M.; Wu, P.H.; Pan, M.; et al. Dual band visible metamaterial absorbers based on four identical ring patches. Phys. E: Low-Dimens. Syst. Nanostruct. 2020, 114526. [CrossRef]

21. Cen, C.L.; Chen, Z.Q.; Xu, D.Y.; Jiang, L.Y.; Chen, X.F.; Yi, Z.; Wu, P.H.; Li, G.F.; Yi, Y. High Quality Factor, High Sensitivity Metamaterial Graphene-Perfect Absorber Based on Critical Coupling Theory and Impedance Matching. Nanomaterials 2020, 10, 95. [CrossRef] 
22. Chen, Z.; Liu, L.; He, Y.; Ma, H. Resolution enhancement of surface plasmon resonance sensors with spectral interrogation: Resonant wavelength considerations. Appl. Opt. 2016, 55, 884-891. [CrossRef] [PubMed]

23. Maharana, P.K.; Srivastava, T.; Jha, R. On the performance of highly sensitive and accurate graphene-on-aluminum and siliconbased SPR biosensor for visible and near infrared. Plasmonics 2014, 9, 1113-1120. [CrossRef]

24. Caucheteur, C.; Shevchenko, Y.; Shao, L.; Wuilpart, M.; Albert, J. High resolution interrogation of tilted fiber grating SPR sen-sors from polarization properties measurement. Opt. Express 2011, 19, 1656-1664. [CrossRef] [PubMed]

25. Vlček, J.; Pištora, J.; Lesňák, M. Design of Plasmonic-Waveguiding Structures for Sensor Applications. Nanomaterials 2019, 9, 1227. [CrossRef] [PubMed]

26. Cuixia, Z.; Guo, X.; Guodong, W.; Shiqun, J. Effect of Spectral Power Distribution on the Resolution Enhancement in Surface Plasmon Resonance. Photonic Sens. 2018, 8, 310-319.

27. Ouyang, Q.; Zeng, S.; Jiang, L.; Hong, L.; Xu, G.; Dinh, X.Q.; Qian, J.; He, S.; Qu, J.; Coquet, P.; et al. Sensitivity Enhancement of Transition Metal Dichalcogenides/Silicon Nanostructure-based Surface Plasmon Resonance Biosensor. Sci. Rep. 2016, 6, 28190. [CrossRef]

28. Xia, G.; Zhou, C.; Jin, S.; Huang, C.; Xing, J.; Liu, Z. Sensitivity Enhancement of Two-Dimensional Materials Based on Genetic Optimization in Surface Plasmon Resonance. Sensors 2019, 19, 1198. [CrossRef]

29. Chan, H.; Guo, X.; Shiqun, J.; Mingyong, H.; Su, W.; Jinyu, X. Denoising analysis of compact CCD-based spectrometer. Optik 2018, 157, 693-706.

30. Homola, J. On the sensitivity of surface plasmon resonance sensors with spectral interrogation. Sens. Actuators B Chem. 1997, 41, 207-211. [CrossRef]

31. Rahman, M.S.; Anower, S.; Hasan, R.; Hossain, B.; Haque, I. Design and numerical analysis of highly sensitive Au-MoS 2 -graphene based hybrid surface plasmon resonance biosensor. Opt. Commun. 2017, 396, 36-43. [CrossRef] 